Short Communication

\title{
Label-Free Electrochemical Detection of MicroRNAs via Intercalation of Hemin into the DNA/RNA Hybridization
}

\author{
Xinyao Yi ${ }^{1, *}$, Zhixuan $\mathrm{Lu}^{1}$, Yu Kong ${ }^{1}$, Zhuo Chen ${ }^{2, *}$ \\ ${ }^{1}$ School of Materials Science and Engineering, Central South University, Changsha, Hunan, P. R. \\ China 410083 \\ ${ }^{2}$ State Key Laboratory of High Performance Complex Manufacturing, Central South University, \\ Changsha, Hunan, P. R. China 410083 \\ *E-mail: yixinyao@csu.edu.cn, zhuochen@csu.edu.cn
}

doi: $10.20964 / 2017.04 .33$

Received: 15 January 2017 / Accepted: 28 February 2017 / Published: 12 March 2017

\begin{abstract}
MicroRNAs (miRNAs) have been emerged as new biomarkers for the detection of a wide variety of cancers. On the basis of the employment of hemin as electron transfer mediator and signal unit, a simple electrochemical assay for sensitive and selective determination of miRNA has been developed. In the presence of miRNA, hemin can intercalate into the hybridization between the preimmobilized DNA probe and miRNA, thus lead to a reasonable electrochemical signal. In the absence of miRNA, hemin cannot intercalate into the single strand DNA probe which lead to undetectable signal. The current is proportional to the concentration of miRNA in a range between $0.1 \mu \mathrm{M}$ and $1 \mu \mathrm{M}$. The proposed assay is label-free, simple and highly selective toward the target miRNA and could be an alternative for detection of miRNA in clinic samples.
\end{abstract}

Keywords: Electrochemistry; MicroRNAs; Hemin; Label-free

\section{$\underline{\text { FULL TEXT }}$}

(C) 2017 The Authors. Published by ESG (www.electrochemsci.org). This article is an open access article distributed under the terms and conditions of the Creative Commons Attribution license (http://creativecommons.org/licenses/by/4.0/). 